\title{
THE ROLE OF BACKGROUND MUSIC IN THE EXPERIENCE OF WATCHING YOUTUBE VIDEOS ABOUT DEATH AND DYING
}

\author{
Panagiotis Pentaris \\ Department of Social, Therapeutic and Community Studies, \\ Goldsmiths University of London, London SE14 6NW, UK \\ E-mail address: p.pentaris@gold.ac.uk \\ Maria Yerosimou \\ @) $(0 \Theta \Theta$ \\ Department of Music, \\ Goldsmiths University of London, London SE14 6NW, UK \\ E-mail address: mup01my@gold.ac.uk
}

\begin{abstract}
YouTube is the largest video sharing site live at the moment. It has been used to communicate a vast array of information, while it allows for user-generated content. This paper will focus on YouTube videos that communicate death, and in particular will present findings from a preliminary study undertaken by the authors considering the role that background music plays in these videos.

Specifically, this study explores the experiences of the viewers of death-related YouTube videos with and without background music while it makes comparisons in relation to the impact that music has on the viewers' emotional experiences.

We conclude that background music elicits emotions and enhances feelings of sadness and sympathy in relation to the visual content of videos while recommendations for future research are made.
\end{abstract}

Keywords: Background music; death; memorial videos; grief; YouTube.

\section{INTRODUCTION}

Media and communication technologies have long been used for information and knowledge exchange. Various themes and subjects associated with social life have been covered by the media. Such coverage crosses geographical boundaries while the audience remains faithful to perceptions which are shaped by personal characteristics and past personal experiences (Gibson, 2007). In particular, media has been used extensively to communicate death, dying, and bereavement; a touchy but yet approachable topic in the online community (Pentaris, 2014; Field, \& Walter, 2003).

This article focuses on YouTube, a well renowned online platform that not only circulates pieces of information to the public, but also allows the public to generate 
content (Chang, Dale, \& Liu, 2007). The user-generated content that goes hand-in-hand with the usage of YouTube reflects upon a number of themes, ethics, limitations, individual decision-making, to name a few. The focus, however, is threefold and correlational. YouTube has been used for the communication of death and dying since it was launched in 2005 (also see Mosco, 2004). Further, the information that is communicated in the videos is commonly accompanied by music, an element that usually acts as a third dimension in the visual screen, which is usually two-dimensional (Cohen, 1999). In order to gain further insight and expand our understanding of how background music influences the experiences of the viewers' of videos with such content, it is only natural that we seek to understand first the role that background music plays in that experience.

Prior to uploading information on YouTube people rely on everyday experiences in the physical environment in order to familiarise themselves with issues or concerns, or even learn new knowledge, skills or abilities. Death and dying are one particular area, communicated via YouTube videos, that raises a number of questions. Some cover issues such as how neutral is the information that is shared, in terms of influence on the viewer? Why do individual users choose this medium in order to communicate their loss, their feelings about the loss in question, or even to have a memorial tribute via YouTube? Also, how do users choose the piece of music that will accompany their videos and how does it affect the way they will present their message? These are only a few of the questions that arise, mostly referring to the user who is generating and uploading the content. What about the viewers though? What are the important questions to ask in relation to the viewer's experience when watching YouTube videos related to death and dying?

In this paper, the authors focus on background music accompanying the video content, and the roles that plays in the experiences of the viewers' when watching death and dying related YouTube video content. In this account, the authors have appreciated the touchy subject of the video content and are exploring its connection with music; whether it elicits emotions, enhances the experience and in what way, or whether meaning-making, from a viewer's point of view, changes according to the music.

\section{BACKGROUND IN DEATH STUDIES}

Dearth of research in the area makes for a challenging question to answer. Scholars have focused on death and the role of media (Christensen, \& Sandvik, 2014), the impact of online platforms in grieving (Pentaris, 2014; Gibson 2007), and the presence of death in the media (Field, \& Walter 2003). Overlooking the binary of private and public, Lange (2007) suggests that both exist in each other while the mediation of a personal loss becomes public, and the public perception of the loss becomes private.

According to Dorthe Christensen and Kjetil Sandvik (2014, p.1), 'media enable us to establish, maintain and develop reactions with the dead without being 
present in the same space-time continuum. Media enable us to obtain a sense of intimacy and co-presence while at the same time maintaining distance and co-absence. Media are materialities that allow us to communicate with the dead or about the dead over the gaps between the world of the living and whatever spatial and temporal sphere the dead may reside in without being absorbed into these as ourselves'. It is in these terms that the communication of death and dying via YouTube videos enables the living to reconnect or reminisce on the dead and bridge gaps in their previous relational experiences. YouTube 'offers an alternative culture of commemoration where private loss finds articulation in video tributes made in memory of loved ones...On YouTube...the commemoration is virtual and the posted message not necessarily engraved forever...Web memorials often represent cultural expressions affected by personal trauma (Wahlberg, 2009, p.218). Gibson and Altena (2014) suggest, similarly, that YouTube is one of the digital cases in which the deceased are commemorated; a digital culture for mourning that is subjected to the amateur users who generate and upload material. It is this particular form of mediation of death which lacks extensive evidence (Moser \& Dun, 2014) in order to comprehend better the role that complementary content, such as background music is, has.

\section{BACKGROUND IN MUSIC STUDIES}

The question asked with regard to the role of background music in various cases is not new and although there have been numerous attempts at providing an answer, there is still considerable space for further research focusing on specific topics. However, we can now understand the basic reasons for which music is used as an accompanying element, either consciously or unconsciously, and we can shape hypotheses and build on existing research towards a deeper comprehension of the subject. Music, in association with other content, has been studied among others, as a source of emotion in films (Cohen, 2001; 2000), in relation to rituals and ritualistic practice (Dissanayake, 2006), and as a means for mood swings and emotion elicitation in itself (Garrido, \& Schubert, 2011).

This particular study, as mentioned before, focuses on background music in relation to the communication of death in YouTube videos. In an earlier paper, the authors examined the subject based on existing research, and provided hypotheses which are now tested in the present study. In addition, this earlier paper examined the subject thoroughly focusing on music's ability to convey and elicit emotions, and thus presented the musical aspect of these videos spherically, presenting music in such videos from the creator's view, as well as the receiver's. For the purposes of the present study, the authors now focus on the viewer's point of view: a great number of memorial video tributes make use of background music, which has been chosen by the user who generated the content and uploaded the video in an effort to express privately expressed feelings toward the loss (Pentaris, \& Yerosimou, 2014). These feelings are externalised to the public via background music and viewers experience the elicitation of possibly similar emotions. 


\section{METHODS}

This is a pilot study looking to answer the research question 'what is the role of background music when watching YouTube videos associated with death, dying, and bereavement' while it gathers data in regard to how background music shapes the experience of the viewer in general.

This is a quantitative study with qualitative aspects in it, namely participant observation notes while collecting quantitative data (Bryman, 2012). Recruitment of research participants was done by the snowball technique (Silverman, 2011). An initial cover letter reached the potential participants and an initial meeting with one of the researchers followed. During that meeting, participants had the opportunity to ask any relevant questions and also sign off a consent form for participation to the study.

Research participants watched three selected YouTube videos; one covering bullying and suicide, one concerned with the death of a child, and one about a celebrity death. The participants watched each video without background music first and completed a questionnaire. Thereafter they watched the video with background music playing and were asked to fill in the same questionnaire.

The pilot study engaged with ten participants in total. Six were men and four were women. Four participants were in the age group of 26-35, and the rest were 36-45. All participants spoke English; four as native speakers and for six English was a second language. The cohort of participants was far from hegemonic while five were first generation migrants, and one second generation migrant.

The data were analysed using the statistical package for analysing and comparing quantitative data, i.e. SPSS.

\section{RESULTS}

The results of this pilot study are described by video content while they also concentrate on general findings, comparing emotional reactions to video content based on the presence or not of background music while watching the video.

The data shows that when background music is playing while watching the video, then the audience experiences greater emotional reaction rather than without. Figure 1 depicts the level of emotional reaction with and without background music.

Research participants watched three different YouTube videos with various content. Following are Figures 2, 3, and 4. These illustrate the level of emotional reactions of the participants based on background music. In detail, figure 2 is concerned with a video covering a personal story of bullying and teenage suicide. Participants experienced greater emotions when background music was accompanying the video. Figure 3 shows results drawn from watching a memorial video about a child. In this figure for the emotional reaction was high even without background music. This will be explored in the discussion, nonetheless it is worth noting that this video contained scenes and messages that were powerful to the individuals even without music. The major difference was that background 
music caught further the attention of the research participants when watching. Last, figure 4 depicts the results from watching a memorial tribute to a celebrity in the UK. Often, celebrity deaths and tributes to them are part of the discussion about the communication of death in YouTube.

Fig. 1. Levels of emotional reaction based on background music.

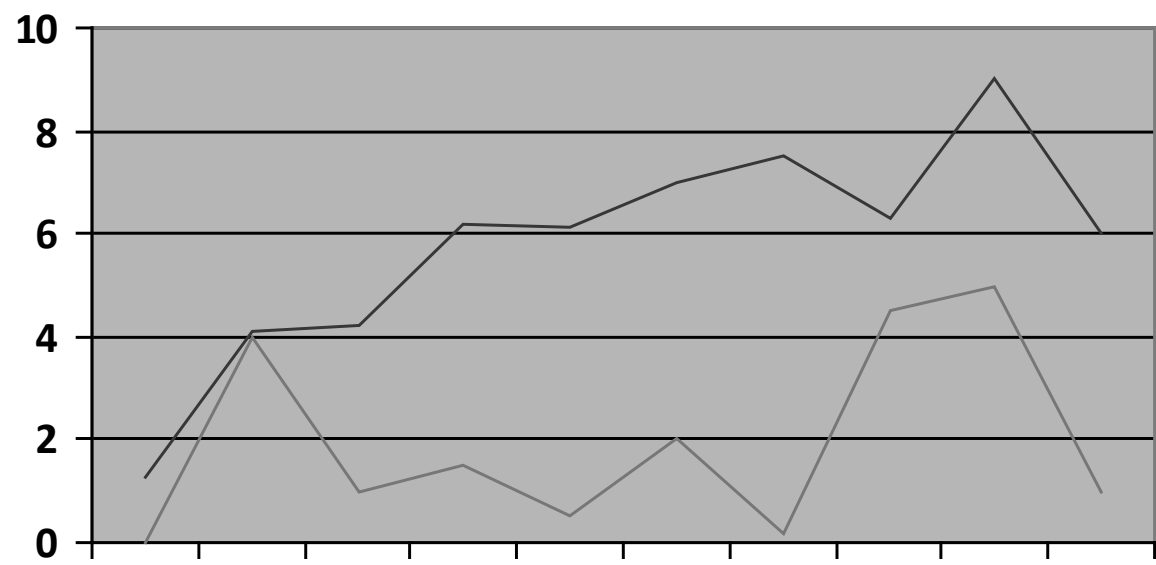

Source: Own chart.

Fig. 2. Emotional reactions based on background music: bullying and suicide.

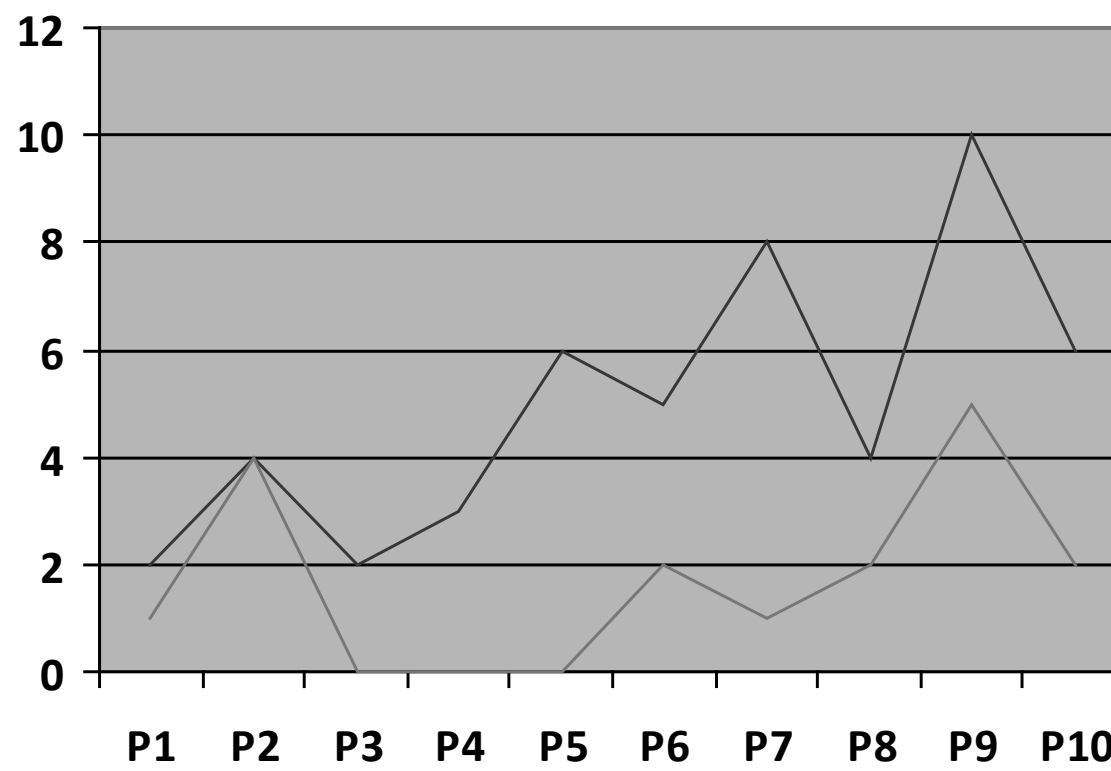

Source: Own chart. 
Fig. 3. Emotional reactions based on background music: death of a child.

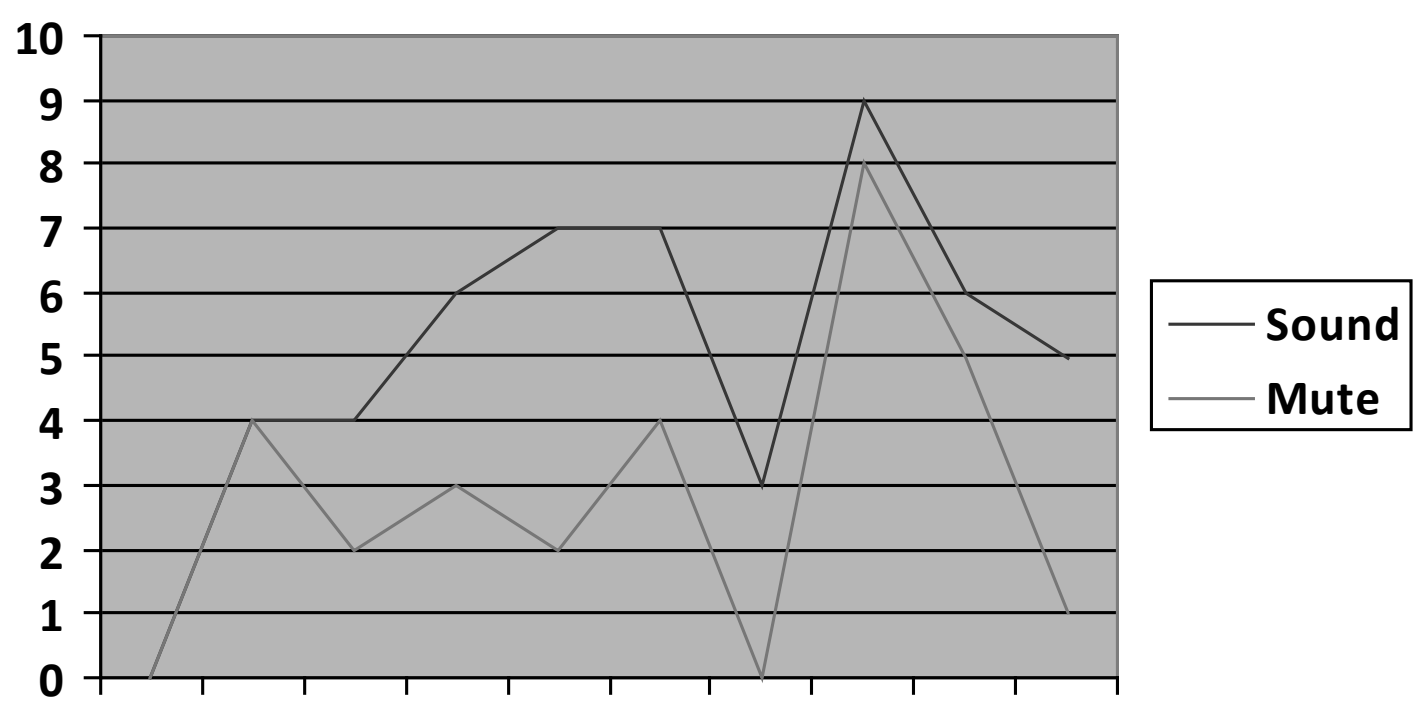

\section{$\begin{array}{llllllllll}\text { P1 } & \text { P2 } & \text { P3 } & \text { P4 } & \text { P5 } & \text { P6 } & \text { P7 } & \text { P8 } & \text { P9 } & \text { P10 }\end{array}$}

Source: Own chart.

Fig. 4. Emotional reactions based on background music: celebrity death.

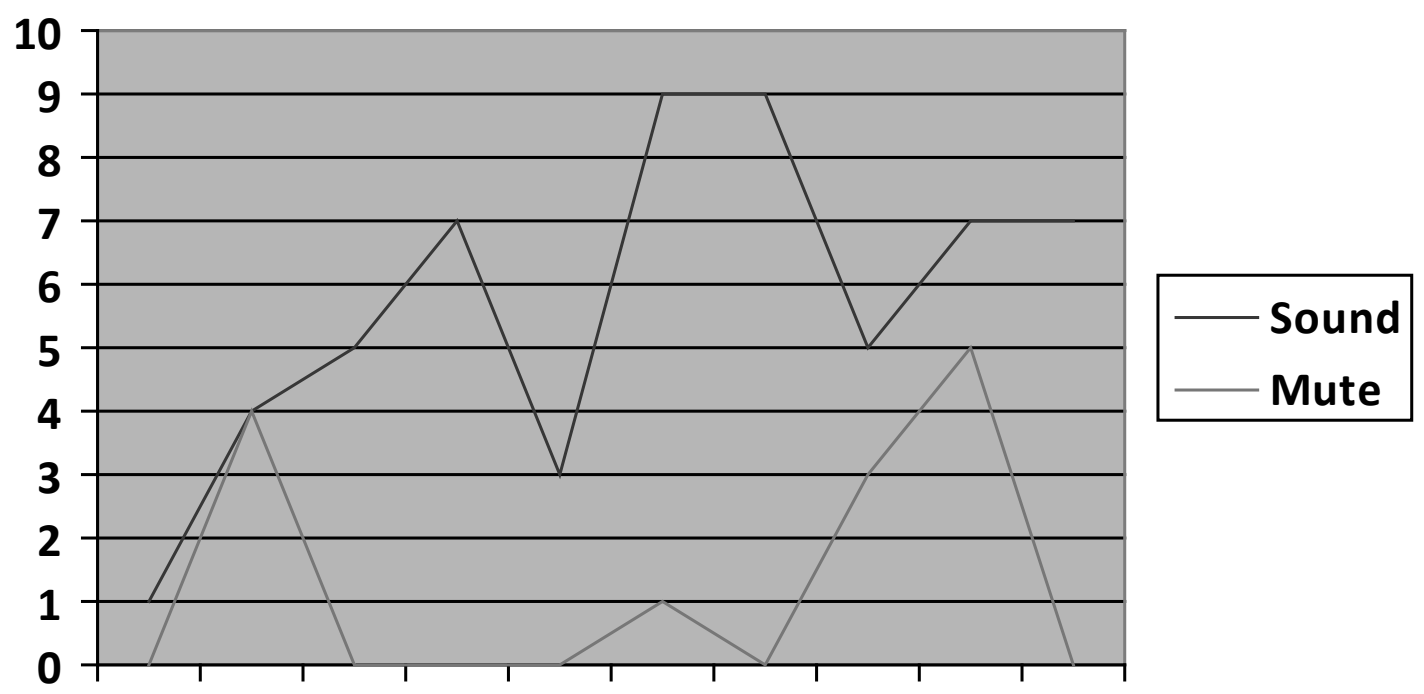

$\begin{array}{llllllllll}\text { P1 } & \text { P2 } & \text { P3 } & \text { P4 } & \text { P5 } & \text { P6 } & \text { P7 } & \text { P8 } & \text { P9 } & \text { P10 }\end{array}$

Source: Own chart.

The research participants were asked to identify their felt emotions during watching the videos, both with and without background music, and later rate the level in which they had experienced them. The following table precisely depicts the emotions that were experienced and identified by the participants. 
Table 1: Various felt emotions experienced during YouTube video watching.

\begin{tabular}{|l|c|c|}
\hline \multicolumn{1}{|c|}{ Felt Emotion } & Without background music & With background music \\
\hline Anger & $67 \%(6.7)$ & $73 \%(7.3)$ \\
\hline Amusement & $17 \%(1.7)$ & $27 \%(2.7)$ \\
\hline Anxiety & $57 \%(5.7)$ & $67 \%(6.7)$ \\
\hline Calm & $82 \%(8.2)$ & $54 \%(5.4)$ \\
\hline Compassion & $63 \%(6.3)$ & $83 \%(8.3)$ \\
\hline Confusion & $40 \%(4)$ & $40 \%(4)$ \\
\hline Contempt & $47 \%(4.7)$ & $63 \%(6.3)$ \\
\hline Disgust & $60 \%(6)$ & $63 \%(6.3)$ \\
\hline Embarrassment & $23 \%(2.3)$ & $23 \%(2.3)$ \\
\hline Empathy & $48 \%(4.8)$ & $77 \%(7.7)$ \\
\hline Excitement & $3 \%(0.3)$ & $7 \%(0.7)$ \\
\hline Fear & $33 \%(3.3)$ & $30 \%(3)$ \\
\hline Guilt & $13 \%(1.3)$ & $10 \%(1)$ \\
\hline Interest & $39 \%(3.9)$ & $63 \%(6.3)$ \\
\hline Intimidation & $30 \%(3)$ & $20 \%(2)$ \\
\hline Sadness & $42 \%(4.2)$ & $97 \%(9.7)$ \\
\hline Shame & $47 \%(4.7)$ & $40 \%(4)$ \\
\hline Shocked & $57 \%(5.7)$ & $63 \%(6.3)$ \\
\hline Sympathy & $50 \%(5)$ & $90 \%(9)$ \\
\hline Surprise & $50 \%(5)$ & $50 \%(5)$ \\
\hline
\end{tabular}

Source: Own table.

The table above highlights the comparison between the level of emotional reaction when the background music was playing and when it was not. In detail, individuals appeared to experience higher levels of sympathy, shock, empathy, contempt, compassion, and anger when the background music was accompanying the video content. In particular, the interest of the participants raised by $24 \%$ when the music was playing. Also, with the background music participants seemed to experience higher levels of anxiety, by $10 \%$, as opposed to being exposed to video content without complementary introductory material.

Another significant finding from the results above is the feeling of being calm. Research participants watching the video content without background music appear to have experienced higher levels of being calm (82\%). On the contrary, background music appears to eliminate this feeling (54\%). Also significant is the feeling of sadness. Research participants experienced great feelings of sadness (97\%) when watching the video content with background music on. However, when the music was not playing, this feeling was diminished by $55 \%$, a significant difference that supports the claim of eliciting emotions.

Figure 5 shows the emotions that were identified and experienced while watching video content without background music. This finding is a correlation between the various video contents. 
Fig. 5. Emotions experienced without background music.

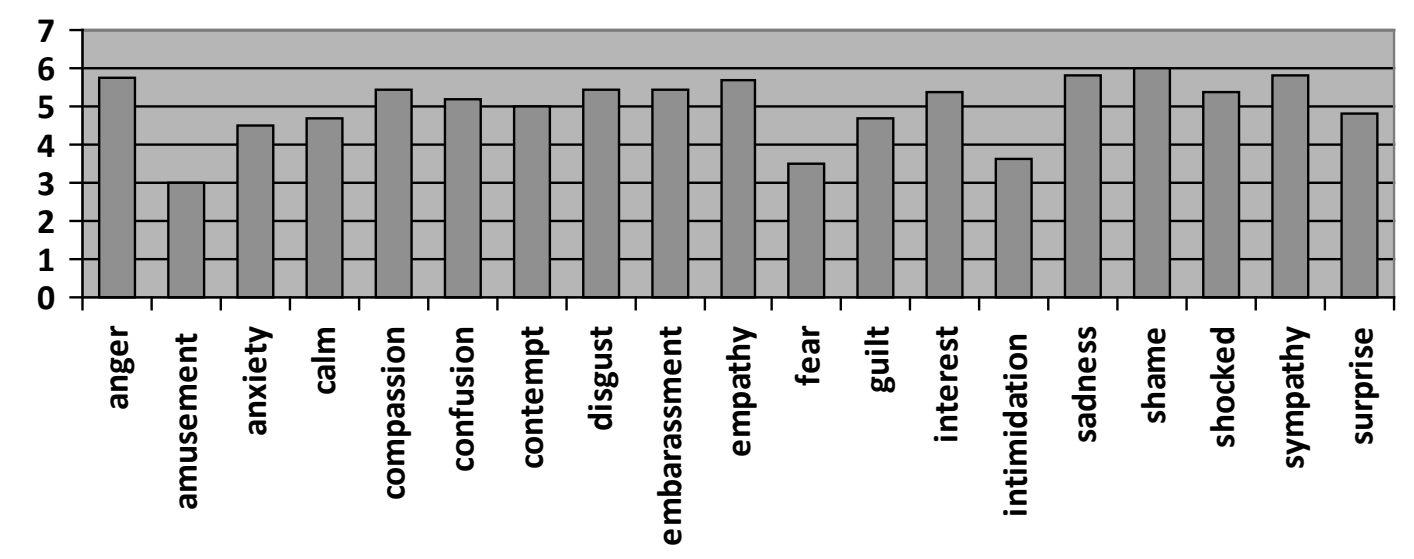

Source: Own chart.

Similarly, figure 6 illustrates the average level to which the identified emotions were experienced during video viewing with background music. The two figures $(5 \& 6)$ depict the significant difference that background music makes when death is communicated via the YouTube platform. What is also worth highlighting here is that different emotions are elicited differently for each category (with or without background music). This, as it will be discussed later, supports further the conversation about generating emotions with supplementary to the video content material.

Fig. 6. Emotions experienced with background music.

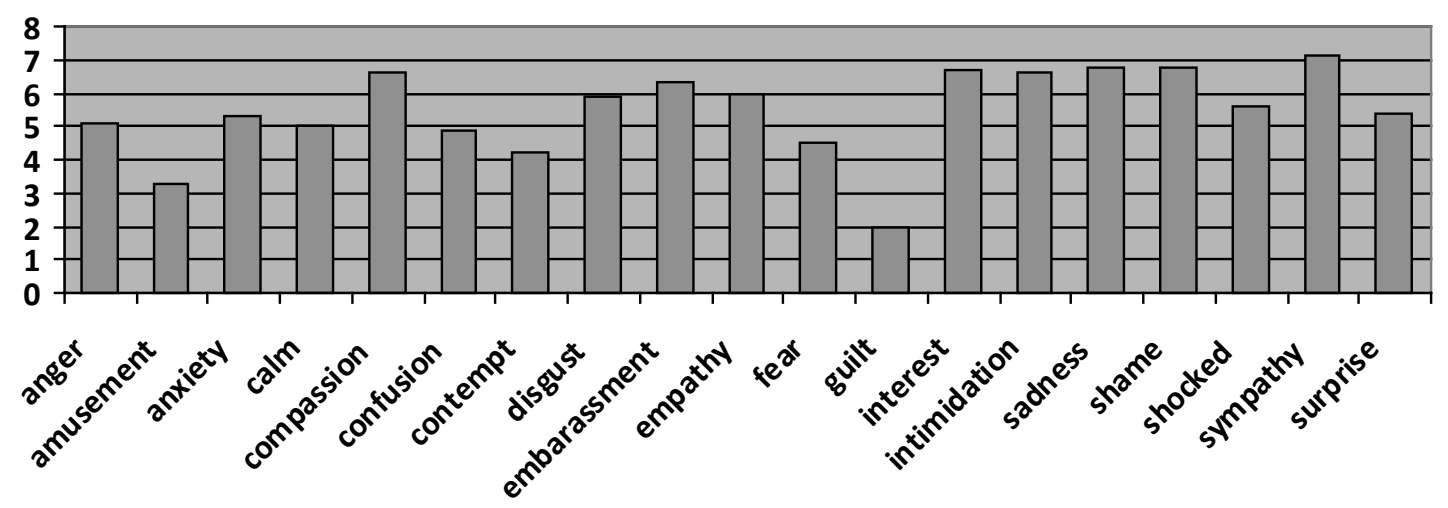

Source: Own chart.

Research participants were asked to indicate how pleasant or not the video content was for them. The following graph illustrates the responses on an average scale. Generally, research participants suggested that the content had been unpleasant regardless of background music. Nonetheless, findings show that when the background music was also playing, the video content was more unpleasant than before. 
Fig. 7. How pleasant was the video content?

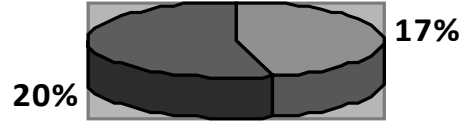

$\square$ With background
music
$\square$ Without
background music

Source: Own chart.

Research participants were also asked to state how engaged or ot they have felt throughout the duration of it. Figure 8 shows that when participants watched the videos with background music, they were engaged more with the content.

Fig. 8. How engaged were you with the video content?

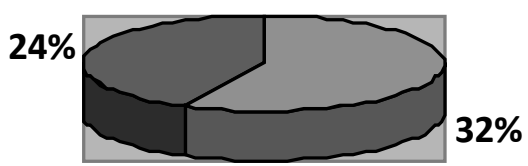

$\square$ With background music

$\square$ Without

background music

Source: Own chart.

\section{DISCUSSION}

It is evident from the results that research participants experienced higher levels of emotional reaction to the video content when that was accompanied by background music. This is paramount in the conversation concerning the functional roles of music, not merely in videos but across a vast array of public and private spaces, whether physical or not. Nonetheless, this study has been concerned with the role of background music when communicating death via YouTube videos. 
Evidence shows that the video content is far from neutral in this occasion. Video sharing on YouTube highlights user-generated content. Therefore, the user has the choice to decide what elements will accompany the video content, i.e. background music. This process is the beginning of making a private experience of dying or grieving open to the public across the globe. Nevertheless, making it public does not necessarily mean that the public will have the luxury to shape their perception around the experience based on their own, neutral from influence, judgement (Pentaris, \& Yerosimou, 2014). The results here show that individuals will experience the video content based on background music. The latter not only enhances previously observed emotions, but it also elicits and generates new emotions that are the result of the music in correlation to the content and not the content per se.

In order to specify when and how background music plays a bigger role, it is important to discuss emotional reactions by each video content. Following, we discuss the three main video content areas that research participants were exposed to for the purposes of this pilot study.

\section{Bullying and suicide}

Emotional reaction when watching the video content with background music is rather intense with a number of emotions, i.e. fear, anxiety, compassion, being predominant in the viewer's experience. This finding is significant when looked at in comparison to the levels of emotional reaction that the video content causes without the background music.

Bullying and suicide are very touchy subjects. However, the viewer of a YouTube video that conveys feelings of grief and loss will not necessarily connect to the content unless there are links with his or her past experiences. If the latter is not the case, then the video content appears to be perceived in a factual, not engaging manner. When background music is added the viewers are generally more engaged with the content while they experience intense emotions that, if they did before, they were to a minimum degree.

Background music in this video content category, also eliminates how pleasant the viewer feels during watching the video. Findings suggest that research participants felt more comfortable and pleasant when watching a video without audio. On the contrary, when the music was playing in the background, this made participants feel unease and intimidated, as well as sad.

\section{Death of a child}

In this category of video content, and in both occasions, i.e. watching the video with and without background music playing, research participants experienced high degree of emotional reactions. Nonetheless, background music affected the experience of the viewers who experienced higher levels of sadness, compassion, anxiety, anger, empathy, interest, shock, and sympathy. In other words, the findings show that watching a video which acts as a memorial tribute to a child that is deceased, is upsetting in itself. However, background music adds to the feelings of anxiety, as well as the ones mentioned above, for the viewers, and it challenges them by increasing fear toward the loss of one's own child, according to the findings of this study. 
Similar to bullying and suicide, background music appears to act as a driving force in the experience of the viewer. It also sets out the context in which the viewer will perceive and comprehend the content of the video. Otherwise, background music may as well be considered as a special physicality in which life experiences occur. Commonly the songs and/or melodies that are chosen for YouTube videos that communicate similar content either have lyrics that are near the idea of loss and love, or function as a calming force toward allowing the viewer to become more available to be affected by the content.

\section{Celebrity deaths}

The communication of celebrity deaths, as well the dissemination of numerous memorial video tributes on YouTube is widely common. Findings of this study demonstrate that once again the viewers experienced a high degree of emotional reactions, namely empathy, anger, calmness, compassion, confusion, sadness, sympathy and shock, when the background music was on.

Similar to the earlier discussion, viewers felt significantly more engaged with the video content when the music was on while in the case of a celebrity death there were also feelings of interest and contempt experienced. Contempt seems relevant to this category because the deceased had been known to the public beforehand, and the viewers might already have shaped an opinion that associates with the deceased and their lifestyle when in life.

\section{FUNCTIONAL ROLES OF BACKGROUND MUSIC WHEN COMMUNICATING DEATH VIA YOUTUBE VIDEOS}

Findings show that viewers experience different emotions differently, all according to the background music that adorns the video content. Previous research in background music suggests that music is another means for communicating information. According to Annabelle Cohen (1999, p.6): “Music is a vehicle transporting a variety of information that serve various multimedia goals. The brain selects what is useful. A prime example of this is the role that emotional meaning from music provided to a visual narrative while sounds of the music are of secondary concern".

Cohen (1999, pp.1-5) suggests that music serves at least eight different functions; 'it masks distraction, provides continuity, directs attention, induces mood, communicates meaning, cues memory, heightens arousal and suspends disbelief and adds an aesthetic dimension'. The following taxonomy of the functional roles of background music in the communication of death via YouTube videos accords with Cohen's suggestion.

While Cohen (1999, pp.1-5.) focuses specifically on film music, we are drawing from empirical research that associates death communication and online platforms. That said, background music appears to draw attention, induce moods and elicit emotions, complement meaning-making process, increases anxiety, and complements engagement with the primary information. Following, we discuss each category in relation to the findings. 
Drawing attention

According to the findings of this study, background music acts as a force for increasing the viewer's interest in the video content, or draw general attention to the content. Data shows that research participants' attention and interest increased due to the background music, which also provided a structure and a sequence in terms of time, keeping their attention to the video and providing a temporal arrangement.

\section{Inducing moods and eliciting negative emotions}

Background music appears to have intense influence on the experiences of the viewers' when watching YouTube videos that relate to death and dying. In particular, background music induces moods in the viewer as those have been defined by Gardner (1985); temporary states that do not directly link to particular behaviour. It is evident that without background music the experience of the viewer is rather different, with less intense feelings and the absence of moods such as sadness or shock. According to Clark and Isen (1982), feeling states are general states and they do not influence on-going behaviour. On the contrary, they act as mood switches to the viewer who is holistically affected by them.

Further, background music enhances the experience of making meanings of death and dying when watching YouTube videos with relevant content by eliciting negative emotions, according to the selection of the music. Those include sadness, empathy, sympathy, compassion, shock, anxiety and anger. With this intent, music usually has a slow tempo, falling contour, low pitch and the minor mode, characteristics associated with conveying sadness (Levi 1985 in Cohen 2000; also Trehub, Cohen, \& Guerriero 1985 in Cohen 2000). In this case, death, dying, and bereavement when communicated via YouTube videos, provide an open space for connection with a subject that the viewer may have not experienced in real life. Nevertheless, YouTube provides the opportunity for familiarisation with it from a distance that can safeguard the individual (Pentaris, \& Yerosimou 2014). The elicitation of emotions is paramount when considering how background music influences the viewing of similar video content while it stresses Lange's (2007) thesis about publicly private and privately public. In other words, the background music that accompanies the content about death and dying has been chosen by the user who generated the content. This choice is personal and can only be perceived via online freedom and the perception that the user-generator decides what and why. The latter comes to the viewer's consciousness only after having experienced the elicitation of their emotions during watching the uploaded video.

\section{Complementing meaning-making process}

YouTube videos are incomplete without an audience. They are uploaded and shared for the public to have access to and interact with them. Two types of interaction are identified in this experience; internal interaction and external interaction. External interaction with a particular video or parts of the video would be to leave a comment, like or dislike the video. On the other hand, internal interaction refers to reactions to the video content but without openly admitting to it or communicating it to the public. 
Regardless, each type of interaction involves a meaning-making process. Viewers not only view the content but they also engage with it. Background music increases the degree of engagement while it sketches ways in which the viewer makes meaning of what is viewed. According to Sirius and Clarke (1994, in Cohen 2000), the meaning of a simple moving geometric figure can be altered according to the background music. In other words, background music highly contributes to how the viewer will perceive, understand, and comprehend the video content. For example, the death of a child has a significant effect on viewers as it elicits emotions of sadness, sympathy and anger. When the same content is presented with background music the viewer's experience is enhanced; negative emotions dominate, anxiety is increased and the meaning they make of the loss of a child and possibly how a parent experiences it, is shaped by the music; if not shaped, coloured and intensified.

\section{Increasing anxiety}

Carol Smith and Larry Morris (1977) have concluded that background music increases anxiety more than it induces calmness. However, this category is dependent on the context in which background music is played. Other research has shown (Bolwerk, 1990; Davis, \& Thaut 1989) that music in general acts as a force toward relaxation, especially with anxiety patients (Li et al., 2012). Nevertheless, this study has focused on background music in a specific context. That said, the content of the video, i.e. death, dying, and bereavement related, already sets the mood while the background music shapes the experience toward the general emotions that death and dying related subjects elicit. Therefore, background music here has the role of increasing anxiety rather than enhancing calmness. Individual viewers feel more upset and at unease when the video content that is indolent and depressive often coincides with background music chosen specifically to accompany and highlight this particular content.

\section{Enhancing engagement}

Background music in YouTube videos that communicate death, dying, and bereavement is also found to enhance the degree of engagement of the viewer with the video and its content. This is tightly connected with the aforementioned functions of music concerning the elicitation of emotions. Background music elicits emotions and creates a connection between the viewer and the content of the video, which when watched on its own does not present high levels of emotional reaction. Hence, the content of the video becomes personal, and thus, the engagement of the viewer is increased.

In addition, the engagement of the viewers is enhanced by the ability of music to provide continuity. Most of the memorial videos on YouTube are formed by a sequence of photos with the addition, in some cases, of short video clips, all put together in the form of slide shows. Since there is no 'dramatic' sequence, it is difficult for the viewers to be focused and concentrated without any time cue. Background music provides that cue which creates a structure, and it also 'binds' the pictures and videos together in a holistic result. 


\section{CONCLUSIONS}

This study is the pilot to a larger study that the authors are currently undertaking. These preliminary results help us to understand the roles of background music in communicating death, dying, and bereavement via YouTube videos. This is paramount and adds to the dialogue about online platforms and the communication of death, dying and bereavement via media and communication technologies. It also contributes to the conversation in regard to how background music influences and shapes the experiences of the audience when used in order to complement primary content presented to the public.

This study has shown that background music in videos with content that relates to death, dying, and bereavement acts as an active influencer to the overall experience while it draws the attention of the viewer, it induces moods and elicits emotions, it complements the meaning-making process, it increases anxiety, and it enhances engagement of the viewer with the video content.

Further, it is suggested in this study that further research is necessary in order to understand better how background music influences the perceptions of the viewers about death and dying, and therefore, how that impacts on the communication of death via media and communication technologies.

\section{REFERENCES}

Bolwerk, C. A. L. (1990). Effects of relaxing music on state anxiety in myocardial infarction patients. Critical Care Nursing Quarterly, 13(2), 63-72.

Bryman, A. (2012). Social Research Methods (4th ed.). Oxford: Oxford University Press.

Chang, X., Dale, C., \& Liu, J. (2007). Understanding the characteristics of internet short video sharing: YouTube as a case study. arXiv preprint arXiv:0707.3670.

Christensen, D.F., \& Sandvik, K. (2014). Mediating and remediating death. Burlington, VT: Ashgate.

Clark, M. S., \& Isen, A. M. (1982). Toward understanding the relationship between feeling

states and social behavior. In: A. Hastorf \& A.M. Isen (Eds.), Cognitive social

psychology (pp. 73-108). New York, NY: Elsevier.

Cohen, A.J. (2001). Music as a source of emotion in film. In: P. Juslin, \& J. Sloboda (Eds.), Music and Emotion (pp. 249-272). Oxford: Oxford University Press.

Cohen, A.J. (2000). Film music: perspectives from cognitive psychology. In: J. Buhler, C.

Flinn, \& D. Neumeyer (Eds.), Music and cinema, Music/Culture (pp.360-377). Hanover, NH: University Press of New England.

Cohen, A. J. (1999). The functions of music in multimedia: A cognitive approach. In: S.W. Yi (Ed.), Music, mind, and science (pp. 40-68). Seoul, Korea: Seoul National University Press.

Davis, W. B., \& Thaut, M. H. (1989). The influence of preferred relaxing music on measures of state anxiety, relaxation, and physiological responses. Journal of Music

Therapy, 26(4), 168-187.

Dissanayake, E. (2006). Ritual and Ritualization: Musical means of conveying and shaping emotion in humans and other animals. In: S. Brown, \& U. Voglsten (Eds.), Music and manipulation: on the social uses and social control of music (pp.31-56). New York, NY: Berghahn Books.

Field, D., \& Walter, T. (2003). Death and the media. Mortality Virtual Themed Issue, 1(4), 1-4.

Gardner, M. P. (1985). Mood states and consumer behavior: A critical review. Journal of Consumer research, 12(3), 281-300.

Garrido, S., \& Shubert, E. (2011). Individual differences in the enjoyment of negative emotion in music: a literature review and experiment. Music Perception, 28(3), 279296. 
Gibson, M. (2007). Death and mourning in technologically mediated culture. Health Sociology Review, 16(5), 415-424.

Gibson, M., \& Altena, M. (2014). The digital lives of the dead: YouTube as a practice of cybermourning. In: D. Moser, \& S. Dun (Eds.), A Digital Janus: looking forward, looking back (pp. 15-27). Oxfordshire: Inter-Disciplinary Press.

Lange, P. G. (2007). Publicly private and privately public: Social networking on YouTube. Journal of Computer-Mediated Communication, 13(1), 361-380.

Li, X. M., Zhou, K. N., Yan, H., Wang, D. L., \& Zhang, Y. P. (2012). Effects of music therapy on anxiety of patients with breast cancer after radical mastectomy: a

randomized clinical trial. Journal of advanced nursing, 68(5), 1145-1155.

Mosco, V. (2004). The digital sublime. London and Cambridge: MIT Press.

Moser, D., \& Dun, S. (2014). A Digital Janus: Looking Forward, Looking Back. Oxfordshire: Inter-Disciplinary Press.

Pentaris, P. (2014). Memorial video tribute and the enfranchised grief of a gay widower.

Thanatos, 3(2), 31-44.

Pentaris, P., \& Yerosimou, M. (2014). Communicating death in YouTube videos: The functional role of music. Journal of Education Culture and Society, 5(1), 206-217.

Silverman, D.(2011). Interpreting Qualitative Data: A guide to the principles of qualitative research (4th ed.). London: SAGE.

Smith, C. A., \& Morris, L. W. (1977). Differential effects of stimulative and sedative music on anxiety, concentration, and performance. Psychological Reports, 41(3f), 1047-

1053.

Wahlberg, M. (2009). YouTube commemoration: Private grief and communal consolation. In: P. Snickars, \& P. Vanderau (Eds.), The YouTube Reader (pp. 218-235). Lithuania: Logotipas.

YouTube (2009). In Memory Of Our Little Jack. Retrieved from

https://www.youtube.com/watch?v=1pm_STRdE3M 